\title{
Structure, contraintes lithostatiques et notation des massifs rocheux
}

P. SIRIEYS

13, rue de la République 38000 Grenoble
Les études de stabilité d'ouvrages (ou de versants), ont tendance actuellement à s'appuyer sur une note globale, affectée au massif, sommant (avec des coefficients variables selon les auteurs) ses caractéristiques géologiques et géomécaniques. Cette note n'est pas une caractéristique intrinsèque du terrain, elle dépend de l'orientation de l'ouvrage (ou de la topographie du versant) dans la structure et dans le champ géostatique, son utilisation n'est donc pas sans risque, Elle ne saurait notamment se substituer à une analyse des champs cinématiques concernant les joints actiffs et passifs induits par l'ouvrage (ou le creusement de la vallée).

Mots-clés : notation, structure, joints, anisotropie, contraintes naturelles, activation, champs cinématiques, tectonique.
NDLE: Les discussions sur cet article sont acceptées jusqu'au 30 juin 2002.

\section{Structure, geostatic stresses and rating of rockmasses}

Studies on construction (or slope) stability now tend to rely on an overall mark given to the rockmass range which takes into account its geological and geomechanical characteristics (with ratings that vary according to the authors). This rating is not an inherent characteristic of the terrain as it depends on how the construction is oriented (on the topography of the slope) in the structure and in the geostatic field. That is why using it is not whitout risk. And notably it should not be used as a substitute for analysis of cinematic fields that concern an active and passive joints resulting from the construction (on the digging out of the valley).

Key words : rating, structure, joints, anisotropy, natural stresses, activating, kinematic fields, tectonic. 


\section{Introduction}

Une estimation globale des qualités mécaniques d'un ensemble rocheux est apparue nécessaire aux projeteurs, et lors de la passation de marchés contractuels.

Selon C. Vibert (1997): «L'usage des classifications des massifs rocheux s'étant largement répandu lest apparue la nécessité del faire un bilan des apports de ces classifications à l'estimation des caractéristiques géotechniques d'un massif rocheux. $x$

Selon P. Duffaut (1999) : «Un des rapporteurs généraux [du 9" Congrès international de la SIMR] a noté avec inquiétude que le terrain physique a presque disparu: La notation des massifs rocheux par une échelle unidimensionnelle, apparue dans les années 70, et promue mondialement par les anglophones, continue à obnubiler ses promoteurs, qui "en rajoutent", et à ravir les bureaux d'études, puisqu'îls n'ont plus à réfléchir. »

Une notation des sols de sous-couche des chaussées a déjà été utilisée avec l'indice de groupe, noté IG, se référant à la granulométrie et aux limites d'Alterberg.

Le massif est donc affecté d’une note, désignée ici par N, quelle que soit la classification adoptée, qui peut être:

- l'indice de qualité Q (Barton et al., 1974, Barton et Grimstad, 1994) :

- le RMR ("Rock Mass Ratio") de Z. Beniavski (1973/1976/1989);

- le GSI ("Geological Strenght Index") de Hoek et al. (1997).

A titre d'exemple, le RMR est la somme de plusieurs termes faisant intervenir les caractéristiques de la roche et du massif: $R$ (Résistance en compression simple de la matrice rocheuse), RQD (Rock Quality Designation), ej (espacement des joints), nj (nombre de joints), w (débit d'eau), S (structure):

$$
\mathrm{N}=\mathrm{N}(\mathrm{R})+\mathrm{N}(\mathrm{RQD})+\mathrm{N}(\mathrm{ej})+\mathrm{N}(n \mathrm{n})+\mathrm{N}(\mathrm{w})-\mathrm{N}(\mathrm{S})
$$

Le poids relatif des termes positifs - N(S) étant un terme correctif - est représenté sur la figure 1.

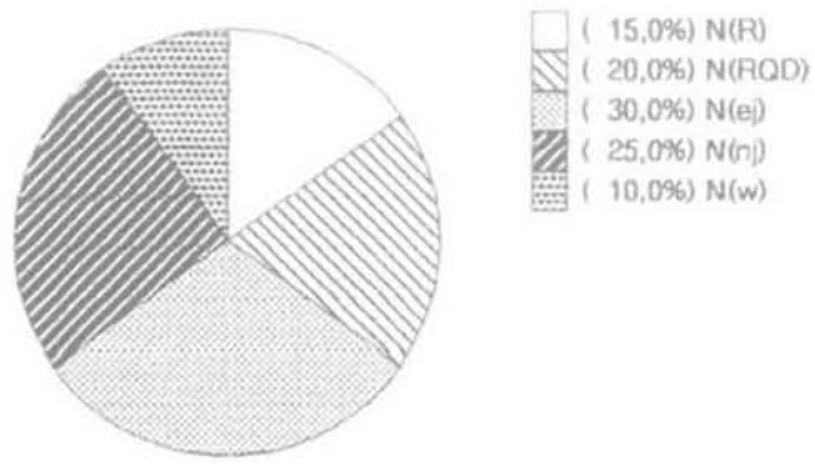

FIG 1 Le RMR (poids des termes). The RMR (relative value of terms).

Une autre méthode d'évaluation, qualitative, consiste (AFTES/Panet et al., 1993, AFTES/Triclot et al., 1999) à affecter aux diverses caractéristiques des massifs, des qualificatifs ( $\alpha$ très faible ) à " très fort » par exemple) dans des classes numérotées (allant de 1 à 3 , indirecte pour chaque caractère envisagé. Ces recommandations de l'AFTES excluent toutefois $\alpha$ d'attribuer au massif une note finale m.

Selon Fookes (1997) cette notation ne présente pas toutes les garanties de fiabilité. Ses tests, qui ont consisté à faire effectuer les mesures sur un mème site, indépendamment, par deux ingénieurs géologues, ont conduit à des valeurs égales à 11 et 62 pour RMR et à 0,022 et 5 pour $Q$, c'est-à-dire pour les deux systèmes d'évaluation, à des écarts très importants induisant, pour le soutènement, des conclusions divergentes.

Les observations suivantes sont destinées à mettre l'accent sur les précautions à prendre (sinon les inconvénients et dangers à éviter) vis-à-vis d'une quantification par un scalaire (N), qui ne saurait ètre considéré comme une caractéristique intrinsèque d'un massif.

\section{2}

\section{Structures}

\section{1}

\section{Les familles structurales}

La structure géologique, à l'échelle d'un projet d'ouvrage ou d'un risque naturel, peut être schématisée, suivant la nature des roches et leur passé (tectonique, altération éventuelle) en quelques grandes familles de surface (souvent planes) dites « familles structurales $)$.

- La stratification $S_{0}$ (ensembles sédimentaires) ou la foliation (surfaces de recristallisation de roches métamorphiques).

- La schistosité S (résultant d'une déformation continue, en tectonique souple).

- Les failles F et diaclasses D ainsi que les fentes d'extension (résultant de déplacements discontinus, selon des modes de rupture distincts, en tectonique cassante).

Ces diverses surfaces de discontinuité sont souvent désignées globalement sous le terme de joints ou discontinuités bien que, selon le dictionnaire de Géologie (Foucault et Raouit, 1984), le terme de joint soit réservé à «Toute surface de discontinuité [...] quil n'est pas un contact avec déplacement $)$.

Ces familles sont des structures discrètes (épaisseur des bancs, espacement des fractures) à l'exception de la schistosité et de la foliation, qui sont des structures dites «pénétratives» (en chaque point du massif passe une surface structurale) donc non-cartographiables.

La structure géologique intervient dans la caractérisation et la stabilité d'un massif, principalement sous trois aspects fondamentaux, pour chaque famille structurale :

- son orientation spatiale;

- sa fréquence:

- ses caractéristiques intrinsèques (ses qualités mècaniques spécifiques, c'est-à-dire ses capacités d'activation ou de réactivation).

Ces trois éléments (non exclusifs) doivent intervenir dans une estimation globale des qualités mécaniques d'un massif rocheux. 


\section{Paramètres d'orientation}

L'orientation de l'ouvrage par rapport à la structure est une caractéristique essentielle à prendre en compte dans l'évaluation géomécanique d'un massif.

\section{4}

\section{Structure monoclinale}

Le creusement d'une tranchée ou d'un tunnel «en direction ) (l'azimut de l'axe de la tranchée, ou du tunnel, coincide avec celui de la structure) pose par exemple des problèmes très différents du cas cen travers bancs $)$ (où ces azimuts font un angle de $90^{\circ}$ ).

\section{a) Études de surface (versants/talus)}

Dans une vallée (Fig. 2), pour le versant «aval-pendage $m\left(a_{1}\right)$ la stratification est active lors d'un glissement banc sur banc, la rupture s'effectue selon une surface $S_{0}$, pour le versant «amont-pendage » $\left(\mathrm{a}_{2}\right)$ la stratification est passive lors d'un glissement de terrain (la rupture est alors dite " astructurale $)$ ).

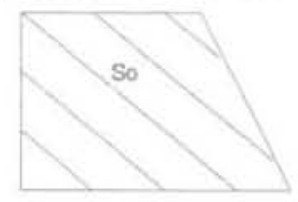

$a_{1}$

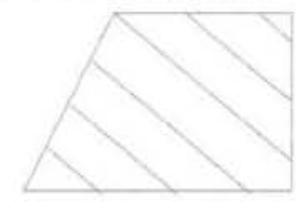

$\mathrm{a}_{2}$

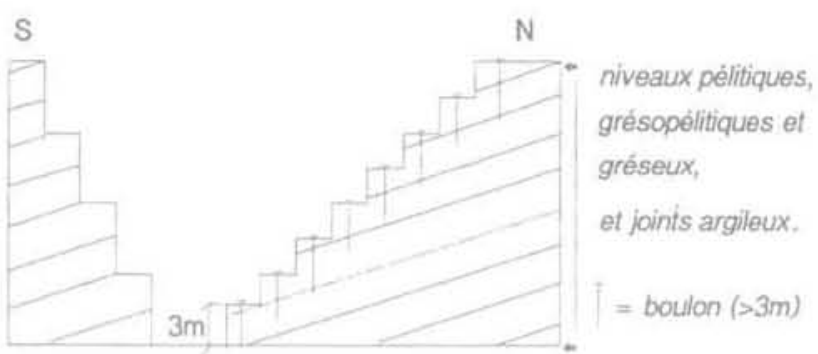

b

FG. 2 Structure monoclinale, 2a: flancs d'une vallée, $\mathrm{S}_{0}=$ stratification $\left(\mathrm{a}_{i}\right.$ : aval-pendage, $\mathrm{a}_{2}$ : amont-pendage), $2 \mathrm{~b}$ : tranchée (à Tréviels, site de Lodève) avec talus à $1 / 1$ et $1 / 2$.

Monoclinal structure, 2a: slide of a valley, $S_{0}=$ stratification $\left(a_{1}\right.$ : downstream-bending. $a_{2}$ : upstream-bending), $2 \mathrm{~b}$; trench (in Tréviels, site of Lodève) with $1 / 1$ and $1 / 2$ bank.

La note $\mathrm{N}$ est pourtant identique dans les deux cas.

\section{Exemple naturel}

Sur un site du bassin de Lodève (Fig. 2b), dans une formation constituée d'une alternance de niveaux décimétriques à demi-métriques de grès pélitiques, de grésopélites et de pélites, d'azimut EW, pentée en moyernne $18^{\circ} \mathrm{S}$, la réalisation d'une tranchée EW a été effectuée en adoptant pour le versant sud (amont-pendage) un talus de pente moyenne $63,5^{\circ}$ (risbermes à 1 pour 2) et pour le versant nord un talus penté de $45^{\circ}$ (1 pour 1) en moyenne. Un glissement s'est produit versant nord, soit au toit d'un niveau pélitique, soit sur un joint argileux, malgré un boulonnage préventif.

\section{b) Travaux souterrains (les tunnels)}

L'axe du tunnel (T), supposé horizontal, est caractérisé par son azimut $\omega_{\mathrm{T}}$, la structure $\mathrm{S}$ (monoclinale), par son azimut $\omega_{\mathrm{S}}$ et son pendage $\beta$ (pouvant être $>0$ ou $<0$ ).

Les différentes configurations conduisent à analyser les quantités:

$\psi=\omega_{\mathrm{T}}-\omega_{\mathrm{S}}$ et $\beta$ (Fig. 3).

Sous réserve d'un état de contrainte naturel peu anisotrope :

Pour $\psi=0$ (tunnel dit $($ en direction $»)$ il est envisageable, dans ce milieu anisotrope, un déplacement axial (la convergence) prédominant en regard du déplacement radial (l'extrusion). (En régime élastique la stabilité serait assurée avec une section elliptique de grand axe normal à S).

Le cas $\beta=0$ est indépendant de $\psi$ (les plans structuraux S étant supposés isotropes).

Pour $\psi=90^{\circ}$ (cas en $($ travers-bancs $\left.)\right)$ : les directions de l'axe du tunnel et de la structure sont orthogonales, des problèmes d'extrusion sont à envisager (d'autant plus importants que $\beta$ est élevé).

Au tunnel de Tartaiguille (André D. et al., 1999) cf. $\$ 3$, la situation est de type: $\psi=90^{\circ}$ et $\beta=10^{\circ}$.

Le RMR tient compte, par son terme négatif, de ces orientations, mais, semble-t-il, de manière insuffisante, notamment pour des pendages faibles.
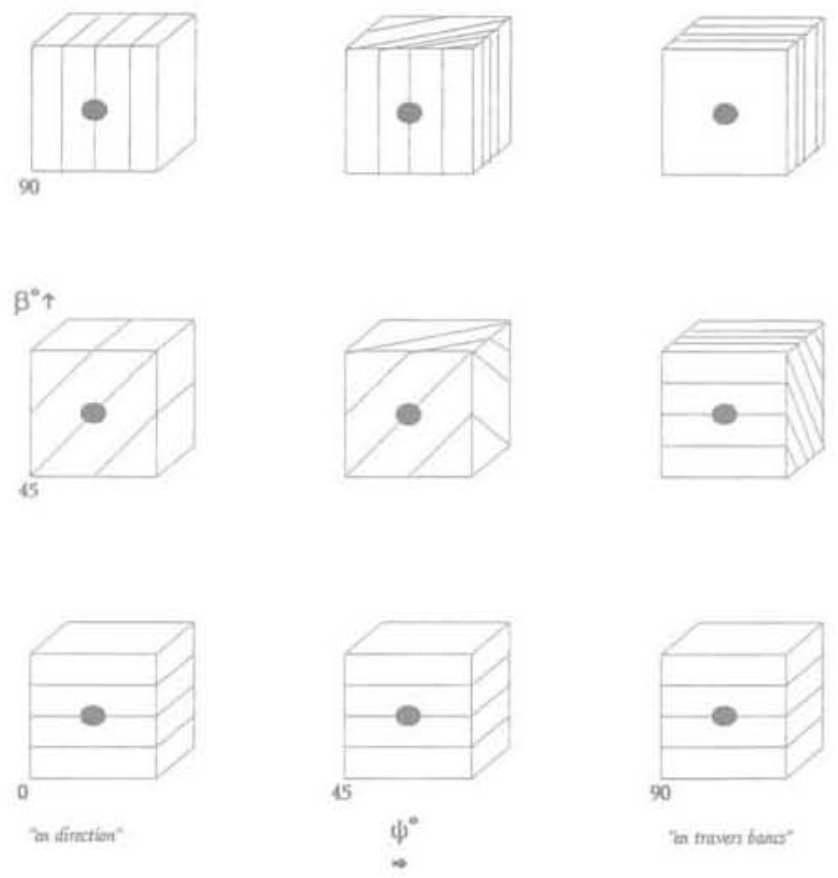

FG. 3 Tunnels: orientation de l'axe par rapport à la structure monoclinale (diagramme $\langle\beta-\psi\rangle$ ), Tunnels: orientation of the axis with respect of the monoclinal structure (diagramm $\langle\beta-\psi>$ ).

\subsection{9}

\section{Structure comportant plusieurs familles de joints}

D'abord, lors de la reconnaissance, les différentes familles de joints sont analysées en orientation (par 
leurs azimuts et pendages, l'ensemble étant souvent représenté en projection stéréographique), Héritées de la déformation antérieure, tectonique, elles s'individualisent généralement assez bien. L'organisation en familles distinctes est la règle générale, puisque résultant du passé tectonique du massif. Plus rarement la représentation est \pm diffuse, \pm homogène, une orientation aléatoire est plutôt l'exception.

Ensuite, ces familles sont hiérarchisées en fonction. d'une part, de leurs caractéristiques mécaniques propres, d'autre part, de leur incidence au niveau du projet.

\section{3}

\section{La fréquence}

Une deuxième caractéristique du terrain est la fréquence de la fracturation, f thomogène à l'inverse d'une longueur), définie, pour une famille, comme linverse de l'espacement moyen, e, de deux joints consécutifs de la famille, soit: $f=1 / e=n / L$. (où $n$ est le nombre de joints sur une distance L normale aux joints, assimilés à des plans). Elle concerne donc les familles discrètes: les joints de stratification (j,) pour lesquels n représente l'épaisseur des bancs, les failles et diaclases $\left(j\right.$, et $\left.j_{d}\right)$ et les joints extensifs $\left(j_{e}\right)$.

\section{Q89,}

\section{Joints de stratification $\left(j_{5}\right)$}

L'assimilation de la stratification à des surfaces planes est acceptable dans les flancs de plis à grande longueur d'onde, ou dans les zones peu tectonisées. Au voisinage des charnières, cette assimilation n'est évidemment plus valable, néanmoins un glissement est possible (exemple du Vaiont, avec une surface de glissement (cen fauteuil»), Muller, 1968).

\section{para \\ Failles et diaclases $\left(j\right.$, et $\left.j_{d}\right)$}

Dans la reconnaissance des failles, une distinction s'impose entre failles "fragiles ») et failles "ductiles ».

Une famille de failles fragiles caractérise une localisation de la déformation uniquement sur des surfaces cisaillantes (souvent planes et d'espacement régulier) dont la fréquence est facilement mesurable.

Une famille de failles ductiles se présente comme des surfaces de discontinuité (des déplacements) nettement individualisées, accompagnées de diaclases (de même orientation) \pm espacées présentant un rejet quasi nul, l'ensemble formant une zone de cisaillement. La déformation naturelle s'est initiée selon un grand nombre de surfaces de cisaillement, de façon quasi homogène, avant de se localiser sur un nombre restreint de failles dites "majeures». Ce mécanisme est observé expérimentalement, en laboratoire, lors d'essais triaxiaux: l'êprouvette montre, en surface, des lignes de glissement (analogues aux lignes de Luders, dans les métaux) avant de se cisailler avec des rejets importants. (L'ensemble caractérise des ruptures en mode 2).

Les mesures de fréquences doivent tenir compte de ces éventualités, le réseau de diaclases, (à rejet infinitésimal) étant associé à la famille de failles « majeures $»$.

\section{$6 x^{2}+3$}

\section{Joints extensifs (je)}

Les joints extensifs (rupture en mode 1) sont caractérisés par un écartement des lèvres de la fracture. Ils ont une forte influence sur la perméabilité du massif. Ils peuvent être remplis de matériau de très faible résistance (argileux) ou, au contraire, “cicatrisés » suite à des circulations de fluides et des cristallisations.

L'observation (Price, 1966) montre que généralement:

$\mathrm{f}_{\mathrm{o}}>\mathrm{f}_{\mathrm{F}}$ (la fréquence des joints extensifs est supérieure à celle des failles).

$\mathrm{f}_{\mathrm{e}}=\mathrm{k} / \mathrm{n}=\mathrm{k} \mathrm{f}_{\mathrm{s}}$ (l'espacement des joints extensifs est proportionnel à l'épaisseur des bancs). croît.

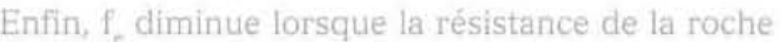

Au tunnel du Siaix (Deville et al., 1993) cette propriété est assez bien vérifiée.

\section{4}

\section{Caractéristiques spécifiques des joints}

L'influence des joints est de deux natures: l'une, géométrique, faisant intervenir la surface occupée par les joints, l'autre, mécanique, relative à leur comportement (mécanique).

La capacité d'activation (ou de réactivation) est la propriété spécifique des structures rocheuses. La striation est particulièrement significative: l'examen d'un plan de faille et l'étude des stries, repérées par leur pitch (angle de la strie avec l'horizontale dans le plan de faille) permettent de définir la direction et le sens du dernier glissement.

\section{1}

\section{Le degré de séparation $(\chi)$}

Le degré de séparation $(\chi)$, défini par: $\chi=s / A$ (avec $\mathrm{S}=$ surface réellement occupée par le joint, $\mathrm{A}=$ surface théorique découpée par le plan de joint) caractérise la discontinuité d'un joint (supposé plan).

Sont utilisés également (AFTES, 1993) : la persistance, qui correspond à la continuité dans l'espace d'une discontinuité, et l'indice de continuité IC, défini par IC = Vlm/VIc (VIm étant la célérité des ondes longitudinales mesurée, Vlc la célérité théorique, calculée à partir de la composition minéralogique).

L'ensemble $f$ et $\chi$ donne une image de la fracturation du massif, $f$ et $\chi$ faibles caractérisant un massif monolithique, $f$ élevé et $\chi=1$ étant l'indice d'un massif très fragmenté, assimilable à la limite à un assemblage de blocs.

\section{Cina?}

\section{Les caractéristiques mécaniques structurales}

Elles concernent l'aptitude au cisaillement (éventuellement à l'écartement) d'un joint. Si la loi de cisaillement sur le joint (valable uniquement sur la surface du joint) est de type linéaire (vérifiant la loi de Mohr-Coulomb), ces caractéristiques (notées $C_{\mathrm{s}}$ et $\phi_{5}$, cohésion 
"structurale» ou "spécifique» et angle de frottement (s structural» ou «spécifique ») vérifient la relation:

$$
\tau_{\mathrm{nt}}=\mathrm{C}_{\mathrm{s}}+\sigma_{\mathrm{n}} \tan \phi \text {, }
$$

( $\tau_{\text {. }}$ et $\sigma_{n}$ étant les composantes tangentielle et normale de la contrainte sur le joint).

Ces valeurs, $C_{\text {s }}$ et $\phi$, difficiles à mesurer, dépendent évidemment de $\chi$, et aussi de l'état de surface du joint: rugosité, matériau intercalaire (surtout dans le cas de joints extensifs), de la direction du glissement antérieur (caractérisée par la strie) notamment dans le cas des joints cisaillants tels que failles ou glissement banc sur banc.

Pour les schistes de Lacaune, les mesures de laboratoire (Saint-Leu et al., 1978) ont fourni pour les plans de schistosité : $\phi_{\mathrm{s}}=22^{\circ}$ et $\mathrm{C}_{\mathrm{s}}=16 \mathrm{MPa}$.

Dans les études de modélisation du massif de Séchilienne, Vengeon (1998, p. 95) utilise dans les codes de calcul, pour les discontinuités : $\mathrm{C}_{\mathrm{s}}=3 \mathrm{MPa}$ et $\phi_{\mathrm{s}}=25^{\circ}$.

Parfois, à défaut de résultats de mesures, la sécurité conduit à prendre $\mathrm{C}_{\mathrm{s}}=0$.

\section{Anisotropie du glissement (sur une surface de joint Sj)}

Le glissement structural dépend du type de joint sur lequel il s'effectue:

- une stratification ( $\mathrm{S}_{0}$ ) passive (non tectonisée), une famille de joints extensifs $\left(J_{0}\right)$ avec remplissage (argileux par exemple) sont assimilées à des surfaces isotropes (milieu orthotrope de révolution);

- une stratification $\left(S^{\prime}\right)$ activée, striée (ayant été le siège de glissements bancs sur bancs), une schistosité (S) ou une foliation présentant une linéation (L), des failles (F) striées sont caractérisées par une anisotropie dans leur plan (milieu orthotrope): $C_{\text {s }}$ et $\phi$, dépendent de l'orientation $\alpha$ du glissement dans le plan: $C_{s}(\alpha)$ et $\phi_{s}(\alpha)$ (Fig. 4).
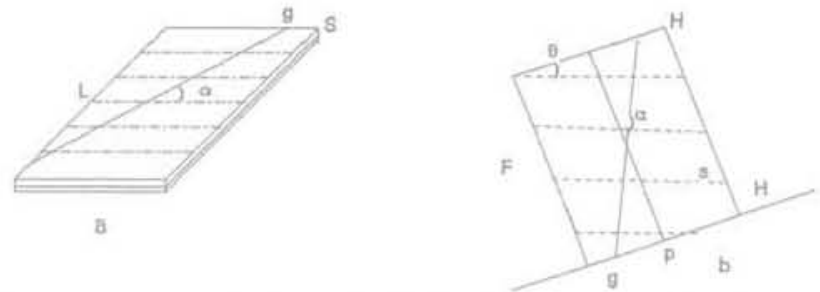

คG. 4 Anisotropie de la résistance au cisaillement dans un joint - 4a : schistosité avec longrain ( $\mathrm{S}=$ schistosité, $\mathrm{L}=$ longrain, $\mathrm{g}=$ direction du glissement, $\alpha=$ angle $\langle\mathrm{L}, \mathrm{g}\rangle$ ) $-4 \mathrm{~b}$ : faille striée $(\mathrm{F}=$ faille, $\mathrm{H}=$ horizontale, $\mathrm{p}=$ ligne de plus grande pente, $\mathrm{s}=$ strie, $\theta=$ pitch, $\mathrm{g}=$ direction du glissement, $\alpha=$ angle $\langle s, g\rangle$ ). Anisotropy of the shearing strength in a joint 4a: schistosity with lineation $(S=$ schistosity, $L=$ lineation, $\mathrm{g}=$ direction of slip, $\alpha=$ angle $<\mathrm{L}, \mathrm{g}>$ $-4 b$ : streaked fault ( $F=$ fault. $H=$ horizontal. $\mathrm{p}=$ the fall of the greater slope, $s=$ streak. $\theta=$ pitch,$g=$ direction of slip, $\alpha=$ angle $\langle s, g\rangle$ ).

Finalement sont envisagés:

- des joints pseudo-isotropes (stratification passive, joints extensif́s \pm colmatés) pour lesquels les constantes structurales dépendent de la rugosité et de la géométrie des surfaces;

- des joints anisotropes (pour lesquels il s'agira de rejeux), d'une part, les foliations et schistosités à anisotropie modérée et, d'autre part, les surfaces striées (stratification activée et failles) sans doute à anisotropie plus forte.

Une autre façon de prendre en compte la structure, dans l'estimation de la résistance d'un massif, consiste à adopter, dans la loi de Hoek et al. (1997) des coeffi- cients minorants tels que ms et a, ce qui revient à remplacer le milieu naturel par un milieu isotrope affecté d'un coefficient de sécurité.

\section{3}

\section{Le champ géostatique et son anisotropie}

La déformation et la stabilité du massif dépendent également de l'état de contraintes naturel, actuel $\left(\Sigma_{0}\right)$, dont la modification (naturelle ou anthropique) induit un champ cinématique (avec éventuelle activation de familles de joints).

$\left(\Sigma_{v}\right)$, qui dépend de l'histoire du massif (notamment de la dernière étape tectonique), fait l'objet de mesures in situ, et aussi d'hypothèses: les plus fréquentes, H1 et H2, concernent son orientation (celle de sont trièdre principal To) et son intensité (caractérisée par ses trois contraintes principales $\sigma_{\gamma}, \sigma_{2}$ et $\sigma_{3}$ ):

- H1: une direction principale est verticale. To ne dépend alors que d'un paramètre, l'angle $\alpha$ dans le plan horizontal,

- H2: la contrainte $\sigma$, principale, est égale à la pression du terrain supérieur (hypothèse dite du «poids de couverture s),

Dans un milieu à surface topographique horizontale l'intensité de $\left(\Sigma_{0}\right)$ est alors caractérisée par un coefficient $K_{0}=\sigma_{0} / \sigma_{2}$, fonction de $\alpha$, soit $K_{0}(\alpha)$. S'il y a isotropie dans le plan horizontal, soit $\sigma_{\gamma}=\sigma, K_{0}$ est un scalaire. Pour un milieu vérifiant la loi de Coulomb (angle de frottement $\phi$ ) $\mathrm{K}_{0}$ est compris entre deux limites notées $\mathrm{Ka}$ et $\mathrm{Kp}$, égales à :

$$
K_{\mathrm{u}}=1 / \mathrm{K}_{\mathrm{p}}=(1-\sin \phi) /(1+\sin \phi)
$$

(soit, pour $\phi=30^{\circ}, 1 / 3$ et 3 ).

Pour une structure monoclinale de pendage $\beta, K_{a}$ et $\mathrm{K}$ dépendent de $\beta$.

Le milieu étant caractérisé par les angles $\phi$, et $\phi$

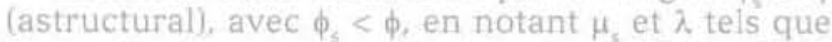
$2 \mu_{s}=\pi / 2-\phi_{s}$ et $\cos 2 \lambda=\sin \phi_{s} / \sin \phi$ le coefficient $K_{s}(\beta)$ s'exprime, en contraintes fictives $(s)=(\sigma)+H(1)$, par: $K_{p}(\beta)=\cot \beta \tan \left(\beta+\phi_{3}\right)$, valable lorsque la structure est active, c'est-à-dire dans la plage de valeur angulaire $2 \lambda$, soit entre $\mu_{s}-\lambda$ et $\mu_{c}+\lambda$ (Sirieys, 1993).

Dans un milieu à surface topographique inclinée (d'un angle i) sur Thorizon, To n'a pas de direction principale verticale.

Seules les mesures in situ permettent une connaissance fiable de $(\Sigma)$.

L'anisotropie du tenseur géostatique peut être estimée à l'aide du tenseur $\left(\Sigma_{0}\right)$ normé en $\sigma_{*}$, soit: $\left(\Sigma_{0}\right) / \sigma_{t}=\left(\mathrm{K}_{0}\right)$ qui est adimensionnel, appelé " pression naturelle réduite) (Une de ses valeurs principales est 1).

Outre l'intensité de ( $\left.\Sigma_{0}\right)$, sa forte anisotropie (Fig. 5) conduit à prendre en considération (indépendamment de la structure) l'orientation de l'ouvrage par rapport à $T \sigma$, le rapport convergence/extrusion en dépend.

Exemples (sur les trois sites suivants $\mathrm{H} 1$ et $\mathrm{H} 2$ sont assez bien vérifiées) :

1) Dans les marnes traversées par le tunnel de Tartaiguille $(\mathrm{T} \sigma)$ est caractérisé par : $\mathrm{X}$ dirigé $\mathrm{EO}$ ( $\alpha$ donc sensiblement perpendiculaire à l'ouvrage n), $\mathrm{Y}$ vertical et $Z$ dans l'axe de l'ouvrage, avec pour $\left(\Sigma_{0}\right): \sigma_{1} / \sigma_{2} / \sigma_{3}=$ $2,5 / 2,1 / 1,8 \mathrm{MPa}$ (André et al., 1999) c'est-à-dire $\mathrm{K}_{0}(\alpha)$ variant de 0,9 à 1,2 . 


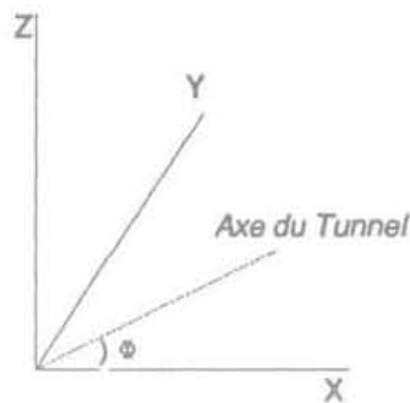

FG. 5 Anisotropie des contraintes naturelles (X. Y = directions principales majeure et mineure dans le plan horizontal, $\phi=\omega \mathrm{T}-\omega \mathrm{X})$.

Anisotropy of natural stresses ( $\mathrm{X}, \mathrm{Y}=$ major and minor principal directions in the horizontal plane, $\phi=\omega \mathrm{T}-\omega \mathrm{X}$.

2) Dans la molasse tertiaire du bassin génevois (constituée d'une alternance de bancs, décimétriques à métriques, faiblement inclinées, de grès, marnes sableuses et marnes, dont la résistance est contrastée: $\mathrm{Rc}>20 \mathrm{MPa}$ pour les grès massifs et de 5 à $7 \mathrm{MPa}$ pour les marnes altérées), $\mathrm{K}_{0}(\alpha)$ est compris entre 1,8 et 2,2 (Laigle et al., 2000).

3) Dans les roches cristallines de la péninsule de Kola, au NW de la Russie et en particulier dans les montagnes de Khibiny (près de Kirovsk), où sont exploités d'importants gisements d'apatite, en carrières et en mines souterraines (To) est tel que $Z\left(\sigma_{3}\right)$ est vertical et $\left(\Sigma_{0}\right)$ a des valeurs proches de : $\sigma_{1} / \sigma_{2} / \sigma_{2}$ $=40 / 20 / 5 \mathrm{MPa}$ pour une profondeur de $200 \mathrm{~m}$ sous la surface du sol (Melnikov et al., 1995; Come, 1999). $K_{0}(\alpha)$ varie donc entre 4 et 8.

\section{Conclusion}

L'ensemble des caractéristiques d'un massif rocheux ne peut être unidimensionnalisé, " scalairisé ». La note attribuée, $\mathrm{N}$, qui somme des éléments résultant de pressions, de longueurs, de nombres, de débits, que mesure-t-elle? que caractérise-t-elle? Un scalaire peut mesurer une caractéristique unique; il ne peut «mesurer $x$ un ensemble de caractéristiques aussi hétérogènes.

Cette note, en outre, n'est pas une caractéristique intrinsèque puisqu'elle dépend de l'ouvrage. L'appréciation d'un massif fait donc intervenir: son passé géologique ayant induit des structures avec leurs paramètres géométriques, ses caractéristiques mécaniques (de déformation, de résistance matricielle et spécifique. de perméabilité), sa pression lithostatique (en valeur moyenne et son anisotropie). Tous ces éléments sont la base de la reconnaissance géologique et géotechnique.

Cette reconnaissance implique une hiérarchisation des joints. Les différentes familles de joints (souvent sont distinguées des familles majeures et mineures) ne jouent pas le même rôle dans la stabilité du massif ; leur hiérarchisation dépend;

- de leurs caractéristiques mécaniques spécifiques, - de leur orientation par rapport à l'ouvrage (angles $\psi$ et $\beta$ ).

Les champs cinématiques (des déplacements et des déformations) résultant du type d'ouvrage, induisent des familles activables et des familles passives. Ce caractère " actif-passif $)$ d'une famille est fonction des paramètres d'orientation $\beta$ et $\psi$. Seuls sont à envisager, au niveau de la rupture, les champs cinématiques compatibles (ce que permet notamment la modélisation).

La hiérarchisation des familles de joints (qui s'impose aussi aux problèmes de versants) dépend donc des joints eux-mêmes, mais aussi de l'ouvrage. C'est finalement le couple "structure-ouvragen qui pourrait, éventuellement, être «noté » ou tout au moins classé. 
Aftes/Panet M. et al. (1993) - " Texte des recommandations pour une description des massif́s rocheux utilè à l'étude de la stabilité des ouvrages souterrains in Supp. au $n^{\circ} 117$ de Tunnels et Ouvrages couterrains (228 p), p. 12-21

Aftes/Triclot J. et al. (1999) - « Rappel de la classification Aftes des massifs rocheux Tumnels et Ouvrages souterrains n 156 èd. Spéctíque, p. 357.

André D. Dardard B. Bouvard A. Carmes J. (1999) - - La traversée des argiles du tunnel de Tartaiguille v. Tunnels et Ouvrages souterrains, $n^{\circ} 153$, éd. Spécifique, p. 181-190.

Barton N.R., Lien R.. Lunde J. (1974) a Engineering classification of rock masses for the design of tunne support w. Rock Mech 6(4), p. 189-239.

Barton N.R., Grimstad E. (1994) - a The Q system following twenty years of appli. cation in NMT Support selection w. Felsbau. In Geomechanics Colloquy, Salz. burg. Austria.

Bleniawski.Z.T. (1973) - "Engineering classification of rock masses jointed $x$. Trans. S. Aft. Inst. Civ, Engrs. 15, p. 335 344.

Bienlawski Z.T. (1976) - "Rock mass classification in rock engineering $\#$. Proc. Symp Exploration for rock engineering. Cape Town, Balkema, 1. p. 97-106.
Bieniawski Z.T. (1989) - " Engineering rock mass classifications ${ }^{\prime}$. New York Wiley.

Come B. (1999) - Stockages en Russie dt Nord-Ouest v. Sẻance technique du CFMR du 01-04-99. Comm. orale (inedit).

Deville E., Massiglia M.. Robert A., Sirieys P. (1993) - a Le tunnel du Siaix: exemple d'analyse géotectonique appliquèe aux travaux souterrains i. Tumnels e: Ouvrages souterrains, $n^{5} 115$, éd. Spécificue, p. 28-33.

Duffaut P. (1999) - is 9 . Congrẻs internatio nal de mécanique des roches n, Géochronique, $\mathrm{n}^{2} 72, \mathrm{p}, 6$

Fookes P.G. (1997) - in Geology for englneers: the geological model, prediction and performance 11. Quateriy Journat of Engineering Geology, 30. p. 293-424.

Foucault A., Raoult J,F. (1984) - i Dictionnaire de géologie w. (2' éd.), Massor $(348 \mathrm{p})$.

Hoek E. Kaiser P.K. Bawden W.F. (1997)"Support of underground excavations in hard rock 3. A.A. Balkema, Rotterdam Brookfieid.

Laigle F., Boymond B., Saive F., Guitton C. (2000) - "Le nouvel accélérateur di CERN. LHC1. Les études et le début des excavations du lot $1 \mathrm{p}$. Tunnels e ouvrages souterrains. $n^{\circ} 157$, éd. Spécifique, p. 33-38.
Melnikov N.N. Konukhin V.P., Kozyrev A.A., Yufin S.A. Barton N. By T.L. (1995) - i Geomechanical aspects of radioactive waste disposal in deep for mations of hard crystalline rocks 3. C.R. 8. Cong. SIMR, vol. 1, p. 59-62.

Muller L. (1968) - « New considerations or the Vaiont slide $x$. Felsmechanik und ingenieur-geologie (Rock Mechanics and Engineering Geology), Vol VL/1-2 Springer-Verlag, p. 1-91.

Price N.J. (1966) - " Fault and joint deve. fopment in brittle and semi-brittle rock w. Pergamon Press. (167 p).

Saint-Leu C.. Lerau J.. Sirieys P. (1978) Mécanismes de rupture des schisteg de Lacaune (Tarn) - Influence de la pression isotrope 0. Bull. Mineral., 101 p. $437-441$.

Sirieys P. (1993) - a Failure criteria of anlsotropic rocks w, C.R. Coll. Intern. CNRS $7^{\circ} 351$ iuin 1983. J.P. Boehler édit. Bal. kêma-Rotterdam, p, 419-425.

Venqeon J.M. (1998) - u Déformation et rupture des versants en terrain métamorphique anisotrope 3 . Thèse univer sitê J. Fourier. Grenoble (186 p)

Vibert C. (1997) - « Apport des classifications de massifs rocheux à l'estimation de leurs caractéristiques géotechniques s. CFMA, Séance du 04/12/97, Comm. orale (inédit). 